\title{
Campus-Wide Floristic Diversity of Medicinal Plants in Indian Institute of Technology-Madras (IIT-M), Chennai
}

\author{
Arumugam Nagarajan1, Saravanan Soorangkattan'2, Kavitha Thangavel3, \\ Boobalan Thulasinathan ${ }^{3}$, Jothi Basu Muthuramalingam ${ }^{2}$, Arun Alagarsamy ${ }^{3 *}$ \\ ${ }^{1}$ Under Graduate Lab, Department of Biotechnology, Bhupath and Jyoti Mehta School of Biosciences, Indian Institute of \\ Technology-Madras, Chennai, India \\ ${ }^{2}$ Department of Botany, School of Life Sciences, Alagappa University, Karaikudi, India \\ ${ }^{3}$ Department of Microbiology, School of Life Sciences, Alagappa University, Karaikudi, India \\ Email: *arunalacha@gmail.com
}

How to cite this paper: Nagarajan, A., Soorangkattan, S., Thangavel, K., Thulasinathan, B., Muthuramalingam, J.B. and Alagarsamy, A. (2017) Campus-Wide Floristic Diversity of Medicinal Plants in Indian Institute of Technology-Madras (IIT-M), Chennai. American Journal of Plant Sciences, 8, 2995-3012.

https://doi.org/10.4236/ajps.2017.812203

Received: September 20, 2017

Accepted: November 7, 2017

Published: November 13, 2017

Copyright $\odot 2017$ by authors and Scientific Research Publishing Inc. This work is licensed under the Creative Commons Attribution International License (CC BY 4.0).

http://creativecommons.org/licenses/by/4.0/

\begin{abstract}
The floristic diversity of plants and their abundance were analyzed in $2.5 \mathrm{~km}$ campus to explore their medical importance by random sampling. The results for plant diversity in IIT-M campus showed nearly 100 species of flowering plants, with genera belonging to nearly 40 families. The most dominant family in the present study is Fabaceae with 15 species (25\%) of the medicinal trees. In addition, the dominant medicinal herbs belong to the families of Acanthaceae, Apocynaceae, Fabaceae and Rubiaceae containing 4 species (12\%) each. The identified medicinal tree and herb are verified with Red data book to explore their conservation status of every identified medicinal trees and herbs. On comparison with the existing IUCN red data list, the conservation status of $73 \%$ of trees and herbs identified in the present study were not assessed. Also, the biodiversity of plants focused on ethano-botanical aspects to reveal their medicinal uses.
\end{abstract}

\section{Keywords}

Medicinal Plants, Medicinal Tree, Plant Diversity, Ethano Botany

\section{Introduction}

Biodiversity plays a key role in the livelihoods of tribes and other people to meet their needs in day-to-day life. The plant kingdom is directly connected with human beings from the beginning of its origination in the universe [1]. India is one of the vast biodiversity-rich countries in the world where the medicinal plants 
have had traditional importance for ages, specifically, in the fields of Ayurveda, Siddha, Unani, and homeopathy. Nearly $75 \%$ of the remedially important plant species grows in almost wild conditions [2]. In the Southern part of India, Indian Institute of Technology-Madras (IIT-M) geographically located with Latitude:: 13.04N, Longitude:: 80.17E, Altitude:: 6 - $16 \mathrm{~m}$ above mean sea level. The campus spans over an area of 236 ha comprising of three major zones-academic, residential and hostel; each acting as a thriving environment for flora and fauna and a wide variety animals and reptiles. A fourth zone identified as Wilderness Zone has been selected for biodiversity assessment. This zone has the richest biodiversity in the Campus. Each zone approximately holds 60 ha area in an uneven configuration or shape. This wilderness zone vegetation is majorly of Southern Thorn Forest type, dominated by cactus followed by Ficus which has significant cultural value. As per the survey, the campus has 432 species of plants were identified/observed (Figure 1).

\section{Materials and Methods}

The institute campus area has a wide distribution of medicinal plants which was made as the main objective of the present study. The distribution of these medicinal plants/tree were identified the aid of taxonomists from Dept of Botany, Alagappa university by referring to authentic literature of the regional flora web database developed by the Prakati Wildlife club of IIT-Madras, Chennai and cross verified with "The flowering plants of Madras city and its immediate neighborhood" [3]. An effective analysis has been performed to identify the available medicinal plants along with their distribution and their medicinal uses.

\subsection{Ethano Botanical Survey}

The ethno botanical survey was carried out by wild life club volunteers of IIT-Madras, between august and September 2016. This study covered total vegetation of the area with emphasis on the ethno botanical hotspots of IIT-Madras campus.

Habitats of over all vegitation

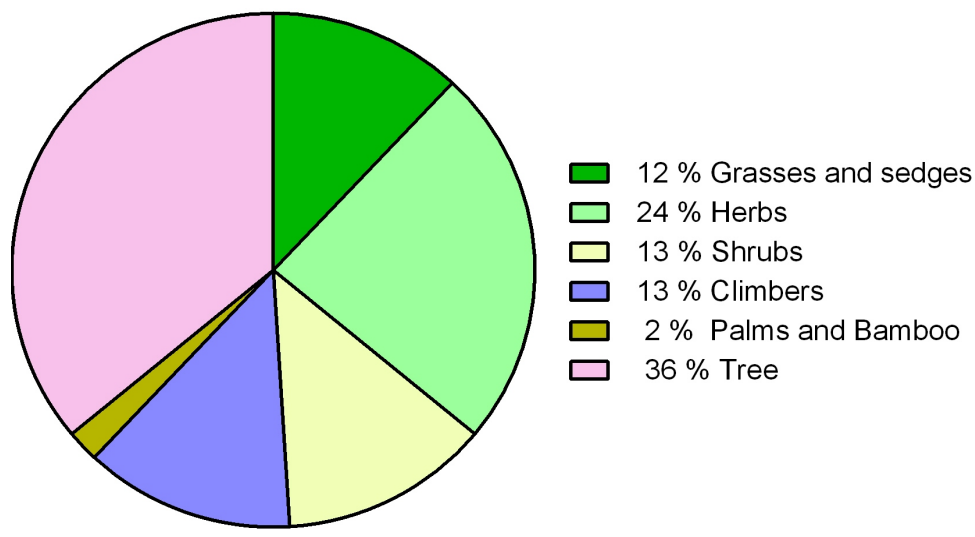

Figure 1. Percentage habitat wise distribution of overall vegetation. 


\subsection{Overall Diversity of Vegetation}

Thirty-six per cent of the non-cultivated plants species are trees. Excluding cultivated trees such as papaya, lime, sapota, etc., that are rarely found outside gardens and homesteads, 107 species of trees are present on the IIT-M campus. The greater presence of tree species has rendered the vegetation type as a forest.

\subsection{Over All Diversity of Medicinal Trees/Herbs}

298 species of non-cultivated plants were identified on the campus. The numbers may increase between 350 - 400 species while including the garden plants. $36 \%$ of the non-cultivated plants on the campus are trees. However, of the trees only $40 \%$ are native. The higher contribution to the diversity of native flora was rose from the grasses (100\%), climbers (84\%) and herbs (80\%). 107 sps., of plants, are non-native. Among these 9 species were invasive. Prosopis juliflora is the most invasive plant on the campus. $50 \%$ to $60 \%$ of the trees in the wilderness zone were dominated by this species. Both tree and herb specimens were collected and identified using regional flora. The collected plant specimen's family and species were confirmed with community herbarium, MS Swaminathan Research Foundation, Chennai.

\subsection{Calculation of Shannon Index of Species Diversity and Species Richness}

The species diversity of medicinal trees and herbs were calculated using Shannon diversity index [4]. It has a combination of two quantifiable measures like species richness and species accountability. Higher in numbers of a species denotes wide range of diversity, Shannon-Wiener diversity Index is defined and given by the following function: MATLAB program was created to calculate the Shannon index. [5].

$$
\begin{aligned}
& \mathrm{H}=\Sigma[(\mathrm{pi}) \times \ln (\mathrm{pi})] \\
& \mathrm{Pi}=\text { proportion of total samples represented by species } \mathrm{i} \\
& \mathrm{S}=\text { number of species, = species richness, } \\
& \mathrm{Hmax}=\mathrm{Ln}(\mathrm{S})=\text { Maximum diversity possible } \\
& \mathrm{E}=\text { Evenness }=\mathrm{H} / \mathrm{Hmax}
\end{aligned}
$$

\section{Determination of Conservation Status of Plants and Herbs}

The universal conservation status of identified trees and Herbs were determined by IUCN red data list cross verification.

\section{Result}

The results on the survey of the plant diversity in the IIT-M campus showed nearly 100 species of flowering plants (both trees and herbs). Among these, 100 genera belonged to 40 families. The most dominant family in the present study was Fabaceae with 18 species (25\%). Next to that, Moraceae comprises 7 species (9\%), Malvaceae include 6 species (8\%). The dominant medicinal herbs are from 
Acanthaceae, Apocynaceae, Fabaceae and Rubiaceae contain 4 species (12\%), and Convolvulaceae, Euphorbiaceae, Phyllanthaceae, and Vitaceae are second dominant with 2 species (4\%) each. The overall habitat is dominated by Trees in the campus (36\%). The detailed investigation of family in the present study and their medicinal values in a different area is represented in Figure 2 and Table 1 \& Table 2.
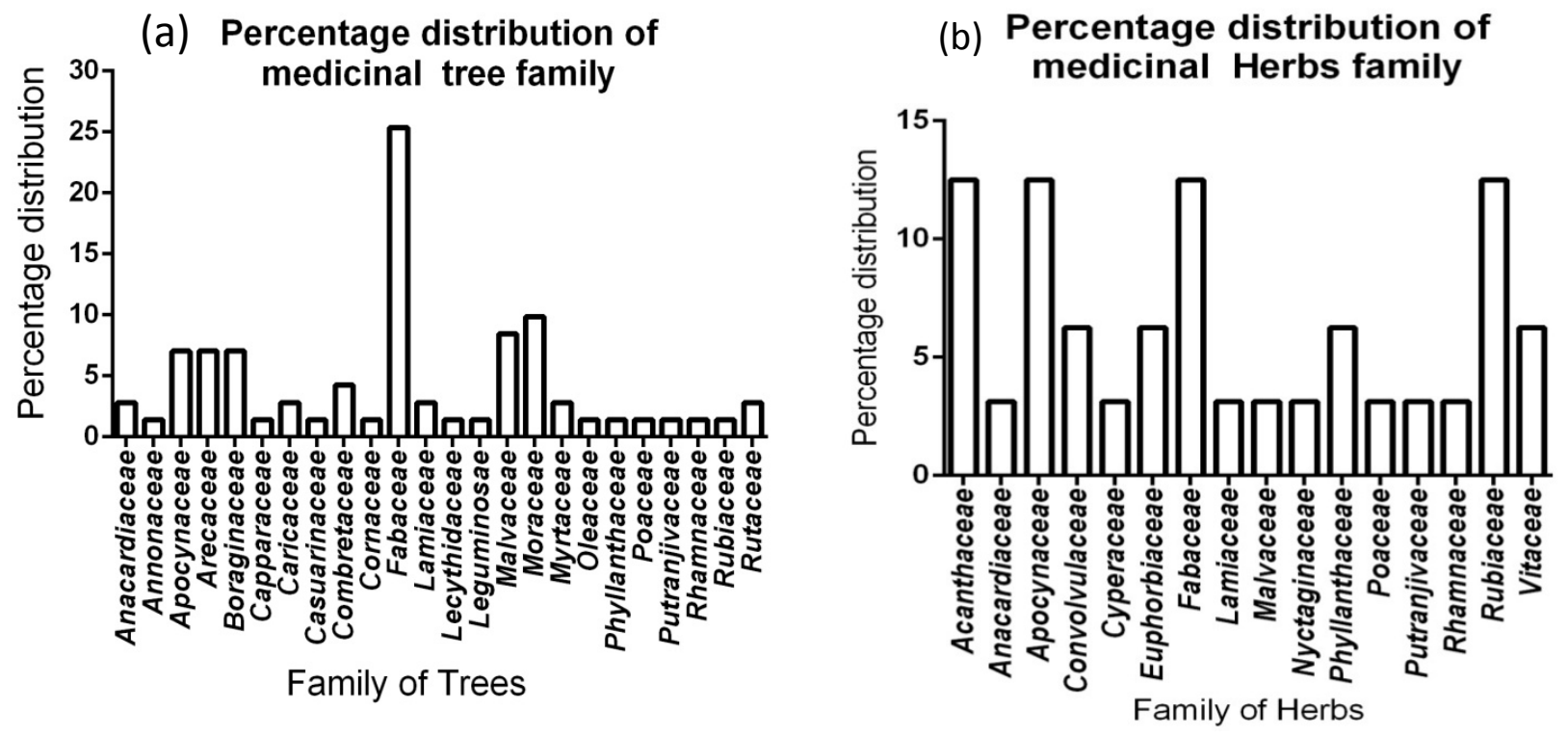

Figure 2. Percentage distribution of (a) medicinal trees and (b) herbs.

Table 1. Diversity of trees on campus.

\begin{tabular}{|c|c|c|c|c|c|c|}
\hline Order & Family & Botanical name & Red data list & Portin of plant & Use & Ref \\
\hline Fabales & Fabaceae & $\begin{array}{c}\text { Acacia } \\
\text { auriculiformis }\end{array}$ & $\mathrm{LC}$ & Bark & Sores and skin complaints & [6] \\
\hline Fabales & Fabaceae & $\begin{array}{c}\text { Acacia } \\
\text { leucophloea }\end{array}$ & $\mathrm{LC}$ & Bark & $\begin{array}{l}\text { Traditional medicine to treat } \\
\text { bronchitis }\end{array}$ & [7] \\
\hline Ericales & Sapotaceae & $\begin{array}{l}\text { Achras } \\
\text { zapota }\end{array}$ & NA & $\begin{array}{l}\text { Premature fruits } \\
\text { and flowers }\end{array}$ & $\begin{array}{l}\text { Treatment of diahheria and } \\
\text { pulmonary complaints }\end{array}$ & [8] \\
\hline Fabales & Fabaceae & $\begin{array}{c}\text { Adenanthera } \\
\text { pavonina }\end{array}$ & NA & $\begin{array}{l}\text { Wood, seed, } \\
\text { leaves and bark }\end{array}$ & $\begin{array}{l}\text { Treatment of rheumatism, } \\
\text { Hair wash }\end{array}$ & [9] \\
\hline Cornales & Cornaceae & $\begin{array}{l}\text { Alangium } \\
\text { salvifolium }\end{array}$ & NA & $\begin{array}{l}\text { Leaves, Fruits, } \\
\text { Tree bark, } \\
\text { Root bark }\end{array}$ & $\begin{array}{l}\text { Anti-diabetic, blood pressure } \\
\text { lowering effect, astringent, rheumatism, } \\
\text { Leprosy and inflammatory patches. }\end{array}$ & [10] \\
\hline Fabales & Fabaceae & $\begin{array}{l}\text { Albizia } \\
\text { lebbeck }\end{array}$ & NA & $\begin{array}{l}\text { Leaves, seeds, } \\
\text { bark, and roots }\end{array}$ & $\begin{array}{l}\text { Gastro intestine complaints Astringent, } \\
\text { anthelmintic and snake bites }\end{array}$ & [11] \\
\hline Gentianales & Apocynaceae & $\begin{array}{l}\text { Alstonia } \\
\text { scholaris }\end{array}$ & LC & $\begin{array}{l}\text { Leaves, bark, } \\
\text { and Fruit }\end{array}$ & $\begin{array}{l}\text { Rheumatic swellings, malarial fevers and } \\
\text { anthelmintic }\end{array}$ & [12] \\
\hline Rosales & Moraceae & $\begin{array}{l}\text { Artocarpus } \\
\text { heterophylla }\end{array}$ & NA & $\begin{array}{l}\text { Leaves, fruit } \\
\text { and seed }\end{array}$ & Skin diseases, astringent, and carminative, & [13] \\
\hline
\end{tabular}




\section{Continued}

\begin{tabular}{|c|c|c|c|c|c|c|}
\hline Sapindales & Rutaceae & $\begin{array}{l}\text { Atlanta } \\
\text { racemosa }\end{array}$ & NA & Stem and leaves & Insect anti feedants & [14] \\
\hline Sapindales & Meliaceae & $\begin{array}{l}\text { Azaridachta } \\
\text { indicia }\end{array}$ & NA & $\begin{array}{l}\text { Leaves, stem bark, } \\
\text { fruits and seed }\end{array}$ & $\begin{array}{l}\text { Anthelmintic, detoxify the blood, treatment } \\
\text { for fever etc., }\end{array}$ & [15] \\
\hline Poales & Poaceae & $\begin{array}{l}\text { Bambus } \\
\text { vulgaris }\end{array}$ & NA & Leaves and shoots & Treatment for febrile disease & [16] \\
\hline Fabales & Fabaceae & $\begin{array}{l}\text { Bauhinia } \\
\text { racemosa }\end{array}$ & NA & $\begin{array}{l}\text { Flower, fruit, root, } \\
\text { leaf. Bark and bud }\end{array}$ & $\begin{array}{l}\text { Oral disorders-cure Toothache-Hunchback- } \\
\text { Burning sensation-Multi nodular Tuberculosis }\end{array}$ & [17] \\
\hline Fabales & Leguminosae & $\begin{array}{l}\text { Bohinia } \\
\text { purpurea }\end{array}$ & NA & $\begin{array}{l}\text { Flower, root, leaf, } \\
\text { bark and bud }\end{array}$ & $\begin{array}{l}\text { Oral disorders, cure Toothache-Hunchback, } \\
\text { Burning sensation, Multi nodular Tuberculosis }\end{array}$ & [18] \\
\hline Malvales & Malvaceae & $\begin{array}{l}\text { Bombax } \\
\text { ceiba }\end{array}$ & NA & $\begin{array}{l}\text { Leaves, root } \\
\text { and flower }\end{array}$ & $\begin{array}{l}\text { Seminal disorders. Nocturnal emission, } \\
\text { semen problems, Leucorrhoea, } \\
\text { Improve breast milk }\end{array}$ & [19] \\
\hline Arecales & Arecaceae & $\begin{array}{l}\text { Borassus } \\
\text { flabellifer }\end{array}$ & NA & $\begin{array}{l}\text { Leaves, root } \\
\text { and flower }\end{array}$ & Antihelmintic and diuretic & [20] \\
\hline Fabales & Fabaceae & $\begin{array}{c}\text { Butea } \\
\text { monosperma }\end{array}$ & NA & Whole plant & $\begin{array}{l}\text { Anti-Semitic, Diarrhea, Dysentery, } \\
\text { Intestinal Worms, Diabetes, Sore Throat }\end{array}$ & [21] \\
\hline Fabales & Fabaceae & $\begin{array}{l}\text { Caesalpinia } \\
\text { coriaria }\end{array}$ & NA & Whole plant & $\begin{array}{l}\text { A sore throat, stomach ache, tonic also used } \\
\text { in tanning purpose in leather processing }\end{array}$ & [22] \\
\hline Brassicales & Capparaceae & $\begin{array}{c}\text { Caparis } \\
\text { zeylanica }\end{array}$ & NA & $\begin{array}{l}\text { Root, seed and } \\
\text { aerial part }\end{array}$ & Antipyretic agent & [23] \\
\hline Brassicales & Caricaceae & $\begin{array}{c}\text { Carica } \\
\text { papaya }\end{array}$ & DD & $\begin{array}{l}\text { Flower, Fruits, } \\
\text { Seed, Leaves, } \\
\text { bark }\end{array}$ & $\begin{array}{l}\text { Carminative, diuretic, chronic diarrhea, } \\
\text { snakebite, Febrifuge, jaundice, pectoral } \\
\text { properties, STD, anti-fungal activity, } \\
\text { jaundice, anti-hemolytic activity, sore teeth. }\end{array}$ & [24] \\
\hline Arecales & Arecaceae & $\begin{array}{l}\text { Caryota } \\
\text { mitis }\end{array}$ & NA & Fruit & Anti-diabetic & [25] \\
\hline Fabales & Fabaceae & $\begin{array}{l}\text { Cassia } \\
\text { fistula }\end{array}$ & NA & $\begin{array}{l}\text { Root, Leave, } \\
\text { bark }\end{array}$ & $\begin{array}{l}\text { Astringent, febrifuge and strong purgative } \\
\text { rheumatism and facial paralysis, insect bites }\end{array}$ & [26] \\
\hline Fabales & Fabaceae & $\begin{array}{l}\text { Cassia } \\
\text { siamea }\end{array}$ & NA & $\begin{array}{l}\text { Fruit, leaves } \\
\text { and flower }\end{array}$ & Antipyretic, to cure intestine worms & \\
\hline Fagales & Casuarinaceae & $\begin{array}{l}\text { Casuarina } \\
\text { equisetifolia }\end{array}$ & NA & Root & Dysentery, diarrhea and stomach ache treatment & [27] \\
\hline Malvales & Malvaceae & $\begin{array}{c}\text { Ceiba } \\
\text { pentandra }\end{array}$ & NA & Leaves & Diabetic reducing agent & [28] \\
\hline Arecales & Arecaceae & $\begin{array}{c}\text { Cocos } \\
\text { nucifera }\end{array}$ & NA & $\begin{array}{l}\text { Coconut shell } \\
\text { fiber Root }\end{array}$ & Diarrhea, renal diseases & [29] \\
\hline Myrtales & Combretaceae & $\begin{array}{l}\text { Combretum } \\
\text { ovalifolium }\end{array}$ & NA & Leaves and root & Antipyretic & {$[30]$} \\
\hline unplaced & Boraginaceae & $\begin{array}{l}\text { Cordia } \\
\text { oblica }\end{array}$ & NA & $\begin{array}{l}\text { Leaves, root, } \\
\text { flower }\end{array}$ & Anti diuretic and antipyretic & [31] \\
\hline Ericales & Lecythidaceae & $\begin{array}{l}\text { Couropita } \\
\text { guianensis }\end{array}$ & NA & $\begin{array}{l}\text { Fruit pulp, bark } \\
\text { and flowers }\end{array}$ & To heal the infected skin on animals, odontalgia & [32] \\
\hline
\end{tabular}




\section{Continued}

\begin{tabular}{|c|c|c|c|c|c|c|}
\hline Fabales & Fabaceae & Delonix regia & LC & Leafs & Antiruhmatism and ant diabetic agent & [33] \\
\hline Fabales & Fabaceae & $\begin{array}{l}\text { Dichrostachys } \\
\text { cinerea }\end{array}$ & LC & Leaf and bark & Vomiting agent & [34] \\
\hline Malpighiales & Phyllanthaceae & $\begin{array}{l}\text { Emblica } \\
\text { Officinalis }\end{array}$ & NA & Leaf, bark & $\begin{array}{l}\text { Immune modulatory, anti-inflammatory, } \\
\text { antiulcer, hepatoprotective, and anticancer } \\
\text { actions. Ant diabetic Ant diarrheal Effect } \\
\text { Analgesic and Antipyretic }\end{array}$ & [35] \\
\hline Fables & Fabaceae & $\begin{array}{l}\text { Enterolobium } \\
\text { contortisiliquum }\end{array}$ & NA & $\begin{array}{l}\text { Root and seed } \\
\text { coat }\end{array}$ & Anti helmintic & [36] \\
\hline Fables & Fabaceae & $\begin{array}{c}\text { Erythrina } \\
\text { variegata }\end{array}$ & LC & Bark, leaves & Antipyretic, asthma and coughs & [37] \\
\hline Rosales & Moraceae & $\begin{array}{l}\text { Ficus } \\
\text { amplissima }\end{array}$ & NA & Bark, leaves & Anti-diabetic, anticancer & [37] \\
\hline Rosales & Moraceae & $\begin{array}{c}\text { Ficus } \\
\text { benghalensis }\end{array}$ & NA & Bark, leaves & Anti-diabetic, Antipyretic, asthma and coughs & [38] \\
\hline Rosales & Moraceae & $\begin{array}{c}\text { Ficus } \\
\text { elastica }\end{array}$ & NA & Bark, leaves & Anti-inflammatory, anti diabetic, anticancer & [39] \\
\hline Rosales & Moraceae & $\begin{array}{c}\text { Ficus } \\
\text { racemosa }\end{array}$ & NA & $\begin{array}{l}\text { Leaves, bark, } \\
\text { latex }\end{array}$ & Anti-diabetes, astringent, astringent. & [40] \\
\hline Rosales & Moraceae & $\begin{array}{l}\text { Ficus } \\
\text { religiosa }\end{array}$ & NA & $\begin{array}{l}\text { Leaves, bark, } \\
\text { latex }\end{array}$ & $\begin{array}{l}\text { Anti helmintic activity, immune modulator } \\
\text { activity, Anticonvulsant activity }\end{array}$ & [41] \\
\hline Lamiales & Lamiaceae & $\begin{array}{c}\text { Gmelina } \\
\text { asiatica }\end{array}$ & NA & $\begin{array}{l}\text { Root, stem bark, } \\
\text { flower and leaf }\end{array}$ & A headache, snake bite, ulcer & [42] \\
\hline Malvales & Malvaceae & $\begin{array}{l}\text { Guazuma } \\
\text { tomentosa }\end{array}$ & NA & $\begin{array}{l}\text { Fruit, Leaf } \\
\text { Root, seed }\end{array}$ & $\begin{array}{l}\text { A cough, Hemorrhage, Infections, Leprosy, } \\
\text { Nephritis, Asthma, Child Birth }\end{array}$ & [43] \\
\hline Ericales & Sapotaceae & $\begin{array}{l}\text { Hibiscus } \\
\text { tiliaceus }\end{array}$ & NA & $\begin{array}{l}\text { Flowers, root } \\
\text { and bark }\end{array}$ & Enema and Anti depressant & [44] \\
\hline Gentianales & Rubiaceae & $\begin{array}{c}\text { Lxora } \\
\text { pavetta }\end{array}$ & NA & Leaves, flower & Anticancer activity & [45] \\
\hline Lamiales & Bignoniaceae & $\begin{array}{l}\text { Kigelia } \\
\text { pinnata }\end{array}$ & NA & $\begin{array}{l}\text { Leaves, fruits } \\
\text { and bark }\end{array}$ & Anti diabetic, Anti inflammatory, anticancer & [46] \\
\hline Sapindales & Rutaceae & $\begin{array}{l}\text { Limonia } \\
\text { acidissima }\end{array}$ & NA & $\begin{array}{l}\text { Fruits, leaves } \\
\text { and root }\end{array}$ & $\begin{array}{l}\text { Astringent, diuretic, cardio tonic } \\
\text { and tonic for the liver and lungs. } \\
\text { The antidote for snake bites. }\end{array}$ & [47] \\
\hline Ericales & Sapotaceae & $\begin{array}{l}\text { Madhuca } \\
\text { longifolia }\end{array}$ & NA & $\begin{array}{l}\text { Flower, fruit } \\
\text { and seed }\end{array}$ & $\begin{array}{l}\text { Analgesic and diuretic. Astringent and } \\
\text { used in chronic tonsillitis and pharyngitis, } \\
\text { rheumatism and skin infection, oil used } \\
\text { in skin disease. }\end{array}$ & [48] \\
\hline Sapindales & Anacardiaceae & $\begin{array}{l}\text { Mangifera } \\
\text { indica }\end{array}$ & $\mathrm{DD}$ & $\begin{array}{l}\text { Leaf, fruit, stem } \\
\text { bark and flower }\end{array}$ & $\begin{array}{l}\text { Anti-diabetic, antiviral activity } \\
\text { Antitumor and HIV, anti diarrheal }\end{array}$ & [49] \\
\hline Fabales & Fabaceae & $\begin{array}{l}\text { Millettia } \\
\text { peguensis }\end{array}$ & $\mathrm{DD}$ & $\begin{array}{l}\text { Leaf, fruit, stem } \\
\text { bark and flower }\end{array}$ & $\begin{array}{l}\text { To treat respiratory difficulties, } \\
\text { Edema, cold and headaches }\end{array}$ & [50] \\
\hline Lamiales & Bignoniaceae & $\begin{array}{l}\text { Millingtonia } \\
\text { hortensis }\end{array}$ & NA & Leaf and flower & Anticancer & [51] \\
\hline
\end{tabular}




\section{Continued}

\begin{tabular}{|c|c|c|c|c|c|c|}
\hline Ericales & Sapotaceae & $\begin{array}{l}\text { Mimusopis } \\
\text { elengi }\end{array}$ & NA & $\begin{array}{l}\text { Leaf, root, fruit, } \\
\text { seed, bark and } \\
\text { flower }\end{array}$ & $\begin{array}{l}\text { Antinociceptive, diuretic effects, gastro } \\
\text { protective, antibacterial, antifungal, anti } \\
\text { cariogenic, free radical anti hyperglycemic }\end{array}$ & {$[52]$} \\
\hline Lamiales & Oleaceae & $\begin{array}{l}\text { Nyctanthes } \\
\text { arbor-tristis }\end{array}$ & NA & Flowers & Anti proliferative and anti diabetic & {$[53]$} \\
\hline Fabales & Fabaceae & $\begin{array}{c}\text { Parkia } \\
\text { biglandulosa }\end{array}$ & NA & $\begin{array}{l}\text { Bark, leaves } \\
\text { and seeds }\end{array}$ & $\begin{array}{l}\text { Dental plaque and caries, pneumonia } \\
\text { stomach ache, severe cough, diarrhea, } \\
\text { wounds, dermatitis, amebiasis, leprosies, } \\
\text { alkalosis, tracheitis, conjunctivitis and } \\
\text { others like hemorrhoids. }\end{array}$ & {$[54]$} \\
\hline Fabales & Fabaceae & $\begin{array}{l}\text { Peltophorum } \\
\text { pterocarpum }\end{array}$ & NA & $\begin{array}{l}\text { Flower, bark } \\
\text { and leaves }\end{array}$ & $\begin{array}{l}\text { Paste of stem bark prepared with water } \\
\text { is applied topically to treat wounds }\end{array}$ & {$[55]$} \\
\hline Arecales & Arecaceae & $\begin{array}{l}\text { Phoenix } \\
\text { sylvestris }\end{array}$ & NA & $\begin{array}{l}\text { Leaf, fruit, } \\
\text { seed and } \\
\text { bark }\end{array}$ & $\begin{array}{l}\text { Insomnia Treatment. Dysentery and } \\
\text { eye lotion }\end{array}$ & {$[56]$} \\
\hline Gentianales & Apocynaceae & $\begin{array}{l}\text { Plumeria } \\
\text { obtusa }\end{array}$ & NA & $\begin{array}{l}\text { Flower and } \\
\text { bark }\end{array}$ & $\begin{array}{l}\text { Antitumor, antiviral, analgesic, } \\
\text { antispasmodic and hypoglycemic }\end{array}$ & {$[57]$} \\
\hline Gentianales & Apocynaceae & $\begin{array}{l}\text { Plumeria } \\
\text { rubra }\end{array}$ & NA & $\begin{array}{l}\text { Leaf and } \\
\text { Pod }\end{array}$ & Abortifacient activity and bio control agent & {$[58]$} \\
\hline Magnoliales & Annonaceae & $\begin{array}{l}\text { Polyalthia } \\
\text { longifolia }\end{array}$ & NA & Bark & $\begin{array}{l}\text { Febrifuge, anti helminthic, skin disease, } \\
\text { diabetes, hypertension, helminthiasis, and } \\
\text { vitiated conditions of vata and pitta }\end{array}$ & {$[59]$} \\
\hline Fabales & Fabaceae & $\begin{array}{l}\text { Pongamia } \\
\text { pinnata }\end{array}$ & LC & $\begin{array}{l}\text { Leaf, bark, seed } \\
\text { and flower }\end{array}$ & $\begin{array}{l}\text { Anti-protozoal, Anti-inflammatory activity, } \\
\text { anticonvulsant activity, Anti-diabetic activity, }\end{array}$ & {$[60]$} \\
\hline Myrtales & Myrtaceae & $\begin{array}{l}\text { Psidium } \\
\text { guajava }\end{array}$ & NA & $\begin{array}{l}\text { Fruits Fruit } \\
\text { skins Leaves }\end{array}$ & $\begin{array}{l}\text { Effect on dental plaque, Antimalarial } \\
\text { effects, Antitussive effects, Hepato } \\
\text { protective effect }\end{array}$ & {$[61]$} \\
\hline Myrtales & Myrtaceae & $\begin{array}{l}\text { Eucalyptus } \\
\text { globulus }\end{array}$ & NA & $\begin{array}{l}\text { Leaves and } \\
\text { bark }\end{array}$ & Head ache anti bacterial activity & {$[62]$} \\
\hline Malvales & Malvaceae & $\begin{array}{l}\text { Pterospermum } \\
\text { acerifolium }\end{array}$ & NA & Flowers & Ant diabetic activity & {$[63]$} \\
\hline Malpighiales & Putranjivaceae & $\begin{array}{l}\text { Putranjiva } \\
\text { roxburghii }\end{array}$ & NA & Seed and fruit & Cough cold and sprue. & {$[64]$} \\
\hline Arecales & Arecaceae & $\begin{array}{l}\text { Roystonia } \\
\text { regia }\end{array}$ & NA & Not yet studied & None & {$[65]$} \\
\hline Fabales & Fabaceae & $\begin{array}{l}\text { Samanea } \\
\text { saman }\end{array}$ & NA & $\begin{array}{l}\text { Leaves, Bark } \\
\text { and root }\end{array}$ & $\begin{array}{l}\text { Treatment for diarrhea, stomachache, } \\
\text { inhibiting effect on Mycobacterium } \\
\text { tuberculosis }\end{array}$ & {$[66]$} \\
\hline Santalales & Santalum & $\begin{array}{l}\text { Santalum } \\
\text { album }\end{array}$ & VU & & $\begin{array}{l}\text { Antiulcer, diuretic, analgesic, antiseptic, } \\
\text { expectorant and stimulant effects }\end{array}$ & {$[67]$} \\
\hline Sapindales & Sapindaceae & $\begin{array}{l}\text { Sapindus } \\
\text { trifoliatus }\end{array}$ & NA & $\begin{array}{l}\text { Leaves and } \\
\text { fruit }\end{array}$ & $\begin{array}{l}\text { Anti dandruff, anti-implantation activity, } \\
\text { eczema, psoriasis, and removing freckles }\end{array}$ & {$[68]$} \\
\hline Fabales & Fabaceae & Saraka asoca & NA & $\begin{array}{l}\text { Leaves and } \\
\text { bark }\end{array}$ & $\begin{array}{l}\text { Antimennorhagic, uterine tonic, } \\
\text { Anti-inflammatory, anti arthritic, cardio } \\
\text { protective effect, Anti-nephrolithiatic }\end{array}$ & [69] \\
\hline
\end{tabular}




\section{Continued}

\begin{tabular}{|c|c|c|c|c|c|c|}
\hline Lamiales & Bignoniaceae & $\begin{array}{l}\text { Spathodea } \\
\text { campanulata }\end{array}$ & NA & $\begin{array}{l}\text { Leaves and } \\
\text { bark }\end{array}$ & Ant malarial, anti-HIV & [70] \\
\hline Malvales & Malveacea & $\begin{array}{l}\text { Sterculia } \\
\text { urens }\end{array}$ & NA & Bark & Aboriginal, bulk laxative, throat infections & [71] \\
\hline Rosales & Moraceae & $\begin{array}{l}\text { Streblus } \\
\text { asper }\end{array}$ & NA & $\begin{array}{l}\text { Root, stem, } \\
\text { bark, Leaves, } \\
\text { seeds }\end{array}$ & $\begin{array}{l}\text { As an application to unhealthy ulcers and } \\
\text { sinuses and as antidote to snake bite, in } \\
\text { epilepsy and obesity }\end{array}$ & [72] \\
\hline Myrtales & Myrtaceae & $\begin{array}{l}\text { Syzygium } \\
\text { cumini }\end{array}$ & NA & $\begin{array}{l}\text { Bark, leaves, } \\
\text { fruits }\end{array}$ & Anti-diabetic, dysentery and bleeding gums & [73] \\
\hline Lamiales & Bignoniaceae & $\begin{array}{l}\text { Tabebuia } \\
\text { aurea }\end{array}$ & NA & $\begin{array}{l}\text { Leaves, bark } \\
\text { and flower }\end{array}$ & $\begin{array}{l}\text { Anticancer, Anti-inflammatory, } \\
\text { Anti malaria }\end{array}$ & [74] \\
\hline Fabales & Fabaceae & $\begin{array}{l}\text { Tamarindus } \\
\text { indica }\end{array}$ & NA & $\begin{array}{l}\text { Leaves, bark } \\
\text { and flower }\end{array}$ & $\begin{array}{l}\text { Laxative, Abdominal pain, diarrhea and } \\
\text { dysentery, Peptic ulcer Antimicrobial, } \\
\text { ant parasitic, antifungal, antiviral, } \\
\text { anti-nematode }\end{array}$ & [75] \\
\hline Lamiales & Lamiaceae & $\begin{array}{l}\text { Tectona } \\
\text { grandis }\end{array}$ & NA & $\begin{array}{l}\text { Leaves, bark } \\
\text { and flower }\end{array}$ & Wound healing activity and dysentery. & [76] \\
\hline Myrtales & Combretaceae & $\begin{array}{l}\text { Terminalia } \\
\text { arjuna }\end{array}$ & NA & Bark & Antioxidant and cardio protective activity & [77] \\
\hline Myrtales & Combretaceae & $\begin{array}{l}\text { Terminalia } \\
\text { catappa }\end{array}$ & NA & Bark & $\begin{array}{l}\text { Wound healing, anti diabetic, anti } \\
\text { oxidant anticancer and anti-mutation }\end{array}$ & [78] \\
\hline Malvales & Malvaceae & $\begin{array}{l}\text { Thespesia } \\
\text { populnea }\end{array}$ & NA & $\begin{array}{l}\text { Leaves and } \\
\text { bark }\end{array}$ & Anti diarrhea, wounds and cholera. & [79] \\
\hline Gentianales & Apocynaceae & $\begin{array}{l}\text { Wrightia } \\
\text { tinctoria }\end{array}$ & LC & $\begin{array}{l}\text { Leaves and } \\
\text { bark }\end{array}$ & $\begin{array}{l}\text { Wound healing activity, Anti-dandruff } \\
\text { activity, Anti-Psoriasis activity }\end{array}$ & [80] \\
\hline Rosales & Rhamnaceae & $\begin{array}{l}\text { Ziziphus } \\
\text { jujuba }\end{array}$ & LC & Fruits & Anorexia, fatigue and dysentery & [81] \\
\hline Sapindales & Anacardiaceae & $\begin{array}{c}\text { Lannea } \\
\text { coromandelica }\end{array}$ & NA & Whole plant & Protozoan infection, AIDS, viral infection & [82] \\
\hline
\end{tabular}

Table 2. Diversity of medicinal plants on campus.

\begin{tabular}{cclcclc}
\hline Order & Family & Botanical name & Red data list & Portion of plant & Use \\
\hline Fabales & Fabaceae & $\begin{array}{l}\text { Alysicarpus } \\
\text { ovalifolius }\end{array}$ & NA & Whole plant & Blood purifier and diuretic \\
Malpighiales & Phyllanthaceae & $\begin{array}{l}\text { Phyllanthus } \\
\text { maderaspatensis }\end{array}$ & NA & Whole plant & $\begin{array}{l}\text { Ant diabetic, spasmodic, wound, } \\
\text { fever, inflammation, antibacterial } \\
\text { and snake bite }\end{array}$ \\
Malpighiales & Violaceae & $\begin{array}{l}\text { Hybanthus } \\
\text { enneaspermus }\end{array}$ & NA & Leaves and fruits & Diuretic, demulcent and tonic \\
Fabales & Fabaceae & $\begin{array}{l}\text { Indigofera } \\
\text { linnaei }\end{array}$ & NA & Whole plant & $\begin{array}{l}\text { Analgesic with anti-inflammatory } \\
\text { activity, }\end{array}$ \\
Poales & Cyperaceae & $\begin{array}{l}\text { Cyperus } \\
\text { rotundus }\end{array}$ & LC & Whole plant & $\begin{array}{l}\text { Spasms, stomach and bowel disorders } \\
\text { and menstrual irregularities }\end{array}$ \\
\hline
\end{tabular}




\section{Continued}

\begin{tabular}{|c|c|c|c|c|c|c|}
\hline Lamiales & Acanthaceae & $\begin{array}{l}\text { Dipteracanthus } \\
\text { prostratus }\end{array}$ & NA & Whole plant & Anti-diabetic & [88] \\
\hline Malvales & Malvaceae & $\begin{array}{l}\text { Corchorus } \\
\text { aestuans }\end{array}$ & NA & Whole plant & $\begin{array}{l}\text { ascites, pain, piles, tumors cystitis, } \\
\text { dysuria, fever, and gonorrhea }\end{array}$ & [89] \\
\hline Caryophyllales & Nyctaginaceae & $\begin{array}{l}\text { Boerhavia } \\
\text { difusa }\end{array}$ & NA & Whole plant & Anti inflammatory & [90] \\
\hline Lamiales & Acanthaceae & $\begin{array}{l}\text { Thunbergia } \\
\text { erecta }\end{array}$ & NA & Whole plant & $\begin{array}{l}\text { Antitumor activity, Cytotoxicity, } \\
\text { Hepato protective activity, } \\
\text { Antinociceptive activity }\end{array}$ & [91] \\
\hline Lamiales & Acanthaceae & $\begin{array}{l}\text { Tylophora } \\
\text { indica }\end{array}$ & NA & Whole plant & $\begin{array}{l}\text { Bronchial asthma, cancer, dysentery, } \\
\text { hay fever, inflammation, rheumatism, } \\
\text { arthritis and dermatitis. }\end{array}$ & [92] \\
\hline Solanales & Convolvulaceae & $\begin{array}{l}\text { Merreria } \\
\text { tridentata }\end{array}$ & NA & Whole plant & Anti-inflammatory and anti arthritis & [93] \\
\hline Gentianales & Apocynaceae & $\begin{array}{l}\text { Ichnocarpus } \\
\text { frutescens }\end{array}$ & NA & Whole plant & Ant diabetic & [94] \\
\hline Solanales & Convolvulaceae & $\begin{array}{l}\text { Evolvulus } \\
\text { nummularius }\end{array}$ & NA & Whole plant & Peptide ulcer & [95] \\
\hline Gentianales & Rubiaceae & $\begin{array}{l}\text { Oldenlata } \\
\text { umbellata }\end{array}$ & NA & Whole plant & Stomach worm & [96] \\
\hline Rosales & Rhamnaceae & $\begin{array}{l}\text { Zyziphus } \\
\text { oenoplia }\end{array}$ & NA & Seeds, leaf & Immuno modulatiory effects & [97] \\
\hline Malpighiales & Putranjivaceae & $\begin{array}{l}\text { Putranjiva } \\
\text { roxburghii }\end{array}$ & NA & Leaves and fruit & Colds, fevers and rheumatism & [98] \\
\hline Malpighiales & Phyllanthaceae & $\begin{array}{l}\text { Phyllanthus } \\
\text { reticulatus }\end{array}$ & NA & Whole plant & $\begin{array}{l}\text { Anti diarrheal, diuretic, cooling } \\
\text { medicine, malaria, asthma and bark } \\
\text { are used as astringent and diuretic. }\end{array}$ & [99] \\
\hline Gentianales & Rubiaceae & $\begin{array}{l}\text { Canthium } \\
\text { dicoccum }\end{array}$ & VU & Whole plant & Anti-diabetic & [100] \\
\hline Gentianales & Rubiaceae & $\begin{array}{l}\text { Benkara } \\
\text { malabarica }\end{array}$ & NA & Bark, leaves & Anticonvulsant activity & [101] \\
\hline Vitales & Vitaceae & $\begin{array}{l}\text { Cissus } \\
\text { vitiginea }\end{array}$ & NA & $\begin{array}{l}\text { Leaves, bark } \\
\text { and root }\end{array}$ & Anti-cholesterol, anti-diabetic & \\
\hline Unplaced & Boraginaceae & $\begin{array}{l}\text { Heliotropium } \\
\text { indicum }\end{array}$ & NA & $\begin{array}{l}\text { Leaves, bark } \\
\text { and root }\end{array}$ & Diarrhea, diabetes, venereal disease & {$[102]$} \\
\hline Lamiales & Lamiaceae & $\begin{array}{l}\text { Hyptis } \\
\text { suaveolens }\end{array}$ & NA & $\begin{array}{l}\text { Leaves, bark } \\
\text { and root }\end{array}$ & Inflammation, gastric ulcer & {$[103]$} \\
\hline Fabales & Fabaceae & $\begin{array}{l}\text { Desmodium } \\
\text { triflorum }\end{array}$ & LC & $\begin{array}{l}\text { Leaves, bark } \\
\text { and root }\end{array}$ & Sedative and anti-tumor & {$[104]$} \\
\hline Malpighiales & Euphorbiaceae & $\begin{array}{l}\text { Croton } \\
\text { bonplandianum }\end{array}$ & NA & $\begin{array}{l}\text { Leaves, bark } \\
\text { and root }\end{array}$ & $\begin{array}{l}\text { Antimicrobial, Anti-inflammatory } \\
\text { Anticancer, Diuretic }\end{array}$ & {$[105]$} \\
\hline Gentianales & Apocynaceae & Carissa sp. & NA & Fruits and leafy & $\begin{array}{l}\text { Headache, rheumatism, rabies } \\
\text { and diuretic }\end{array}$ & [106] \\
\hline Gentianales & Apocynaceae & Vinca rosea & NA & Leaf and flower & $\begin{array}{l}\text { Antihyperglycemic and } \\
\text { hypertensive activity }\end{array}$ & [107] \\
\hline
\end{tabular}

NA: Not yet Assessed; LC: Least Concern; NT: Near Threatened; VE: Vulnerable ; LR: Lower Risk; EN: Endangered; DD: Data Deficient. 
Shannon-Wiener diversity index of species richness $(\mathrm{H})$ for the medicinal tree is 4.263 and evenness $(\mathrm{E})$ is 0.972 , whereas in medicinal herb species richness $(\mathrm{H})$ was calculated as 3.284 and evenness (E) was 0.975 (Figure 3).

In addition to the floristic study, the present study enumerated the medicinally important plants in the study area which are used to cure different diseases. Totally 107 medicinal tree and herbs were identified in the wide campus. Of which $73 \%$ of trees and herbs are not assessed in IUCN red data list (Figure 4).

\section{Conclusion}

The primitive societies of India have been dependent on herbal medicines over a long period of time. In fact, all conventional systems of herbal based therapy had their roots and origin in folk medicines or asthma medicines. The knowledge of ethano botany plays a vital role in the primary health care and economy of tribal's. Increasing populations of our country require the discovery of new herbal drugs to cure both endemic and epidemic diseases without side effects. India has been endowed with rich biodiversity which comprises of nearly $12 \%$ of the global plant wealth. Due to over exploitation of forest and urbanization, one-third of the plants are endemic and endangered. In the present study, various families of

Shanon diversity index of medicinal trees

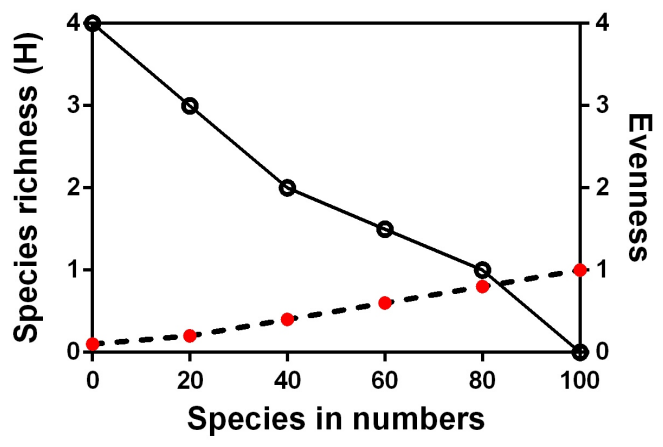

Shanon diversity index of Herbs

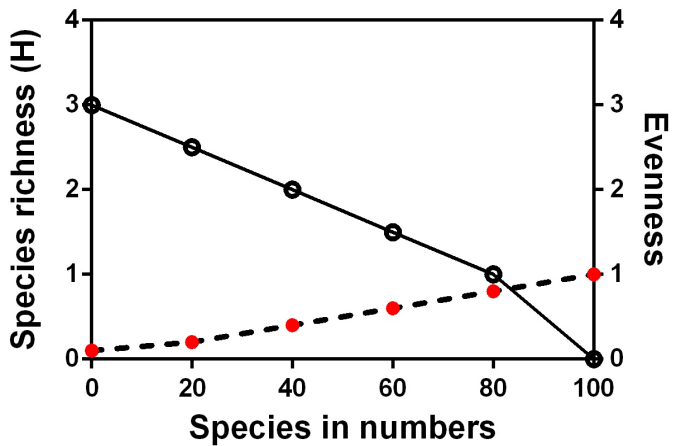

Figure 3. Shannon graph diversity of species richness index of Trees and Herbs in the study area.

Conservation status of Medicinal trees

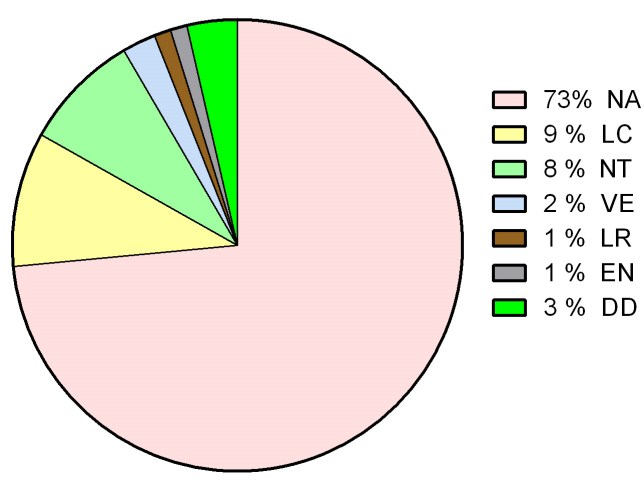

\section{Conservation status of Herbs}

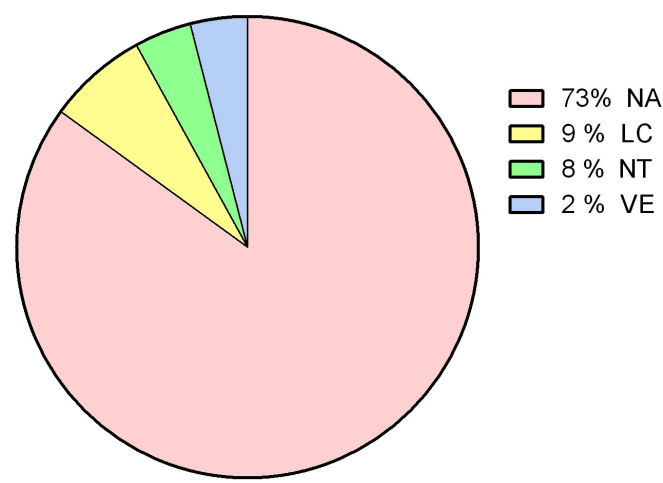

Figure 4. Conservation status of (identified in IITM campus) Tree species reported for medicinal purposes in India according to IUCN Red List. NA: Not yet Assessed; LC: Least Concern; NT: Near Threatened; VE: Vulnerable; LR: Lower Risk; EN: Endangered; DD: Data Deficient. 
flowering plants are identified in the study area (IIT-M campus). Cross verification of identified plants with IUCN Red data book confirmed the IIT-M wide campus is the shelter for nearly threaded (NT), vulnerable (VE) and endangered (ED) species of medicinal trees and herbs.

\section{Acknowledgements}

We would like to express a deep sense of gratitude to Prakrith, Environmental and IIT campus community for his valuable guidance and help. We also extend our thanks to wildlife club awareness team IIT-M online server providers and maintenance authorities for successful completion of this enumeration of plant diversity.

\section{Conflict of Interests}

The authors declare no conflict of interest.

\section{References}

[1] Elizabeth, M. and Dowdeswell, D. (1995) Global Biodiversity Assessment. UNEP, Cambridge University Press, UK, 80-89.

[2] Laloo, R.C., Kharlukhi, L., Jeeva, S. and Mishra, B.P. (2006) Status of Medicinal Plants in the Disturbed and the Undisturbed Sacred Forests of Meghalaya, Northeast India: Population Structure and Regeneration Efficacy of Some Important Tree Species. Current Science, 90, 225-232.

[3] Livingstone, C. and Henry, A.N. (1994) "The Flowering Plants of Madras City and Its Immediate Neighbourhood" by P.V. Mayuranathan, Government Museum, Government of Tamil Nadu, Madras.

[4] Shannon, C.E. and Wiener, W. (1968) The Mathematical Theory of Communication. University of Illinois Press, Urbana, 20-45.

[5] Beck, J. and Schwanghart, W. (2010) Comparing Measures of Species Diversity from Incomplete Inventories: An Update. Methods in Ecology and Evolution, 1, 38-44.

[6] Michael, P. (1990) Ecological Methods for Field and Laboratory Investigation. Tata McGraw-Hill Education Publishing Co. Ltd., New Delhi, 1-50.

http://trove.nla.gov.au/version/231666778

[7] Dabur, R., Gupta, A., Mandal, T.K., Singh, D.D., Bajpai, V., Gurav, A.M. and Lavekar, G.S. (2007) Antimicrobial Activity of Some Indian Medicinal Plants. African Journal of Traditional, Complementary and Alternative Medicines, 4, 313-318. https://doi.org/10.4314/ajtcam.v4i3.31225

[8] Ch, M.I., Khan, M.A. and Hanif, W. (2006) Ethno Veterinary Medicinal Uses of Plants from Samahni Valley Dist. Bhimber, (Azad Kashmir) Pakistan. Asian Journal of Plant Sciences, 5, 390-396. https://doi.org/10.3923/ajps.2006.390.396

[9] Pandhare, R. and Sangameswaran, B. (2012) Extract of Adenanthera pavonina L. Seed Reduces Development of Diabetic Nephropathy in Strep Tozotocin-Induced Diabetic Rats. Avicenna Journal of Phytomedicine, 2.

[10] Suresh, S., Balakrishnan, N.V. and Christudas, S. (2017) Pharmacological and Phytochemical Studies of Alangium salvifolium Wang.-A Review. Bulletin of Faculty of Pharmacy, Cairo University. https://doi.org/10.1016/j.bfopcu.2017.07.001 
[11] Tariq, A., Mussarat, S., Adnan, M., Abd Allah, E.F., Hashem, A., Alqarawi, A.A. and Ullah, R. (2015) Ethnomedicinal Evaluation of Medicinal Plants Used against Gastrointestinal Complaints. BioMed Research International, 1, 1-14. https://doi.org/10.1155/2015/892947

[12] Ganjewala, D. and Gupta, A.K. (2013) Study on Phytochemical Composition, Antibacterial and Antioxidant Properties of Different Parts of Alstonia scholaris Linn. Advanced Pharmaceutical Bulletin, 3, 379-384.

[13] Gupta, N., Jain, U.K. and Pathak, A.K. (2009) Wound Healing Properties of Artocarpus heterophyllus Lam. Ancient Science of Life, 28, 36-37.

[14] Nirmal, S.A., Laware, R.B., Rathi, R.A., Dhasade, V.V. and Kuchekar, B.S. (2011) Antihistamine Effects of Atlantia racemosa Leaves. Journal of Young Pharmacists, 3, 129-131. https://doi.org/10.4103/0975-1483.80301

[15] Kumar, V.S. and Navaratnam, V. (2013) Neem (Azadirachta indica): Prehistory to Contemporary Medicinal Uses to Humankind. Asian Pacific Journal of Tropical Biomedicine, 3, 505-514.

[16] Panee, J. (2015) Potential Medicinal Application and Toxicity Evaluation of Extracts from Bamboo Plants. Journal of Medicinal Plants Research, 9, 681-692. https://doi.org/10.5897/JMPR2014.5657

[17] Reddy, M.H., Reddy, R.V. and Venkata Raju, R.R. (1998) Rutaceous Plants from Tribal Medicine of Andhra Pradesh. Ancient Science of Life, 17, 251-252.

[18] Pahwa, S., Mazumder, R., Bhattacharya, S., Kumari, S., Mazumder, A. and Singh, D.P. (2010) Pharmacognostical and Phytochemical Evaluation of the Leaves of Bauhinia purpurea Linn. Ancient Science of Life, 30, 28-32.

[19] Azam, M.N.K., Rahman, M.M., Biswas, S. and Ahmed, M.N. (2016) Appraisals of Bangladeshi Medicinal Plants Used by Folk Medicine Practitioners in the Prevention and Management of Malignant Neoplastic Diseases. International Scholarly Research Notices, 2016, Article ID: 7832120.

[20] Chaurasiya, A.K., Chakraborty, I. and Saha, J. (2014) Value Addition of Palmyra Palm and Studies on the Storage Life. Journal of Food Science and Technology, 51, 768-773. https://doi.org/10.1007/s13197-011-0561-3

[21] Singh, G., Singh, R., Verma, P.K., Singh, R. and Anand, A. (2015) Anthelmintic Efficacy of Aqueous Extract of Butea monosperma (Lam.) Kuntze against Haemonchus contortus of Sheep and Goats. Journal of Parasitic Diseases, 39, 200-205. https://doi.org/10.1007/s12639-013-0324-3

[22] Jeeva, K., Thiyagarajan, M., Elangovan, V., Geetha, N.P. and Venkatachalam, P. (2014) Caesalpinia coriaria Leaf Extracts Mediated Biosynthesis of Metallic Silver Nanoparticles and Their Antibacterial Activity against Clinically Isolated Pathogens. Industrial Crops and Products, 52, 714-720.

[23] Sini, K.R., Sinha, B.N. and Rajasekaran, A. (2011) Protective Effects of Capparis zeylanica Linn. Leaf Extract on Gastric Lesions in Experimental Animals. Avicenna Journal of Medical Biotechnology, 3, 31-35.

[24] Arumugam, N., Boobalan, T., Raja Rajeswari, P. and Duraimurugan, M.D. (2014) Antimicrobial Activity and Phytochemical Screening of Cynodon dactylon and Carica papaya. Research in Biotechnology, 5, 21-31.

[25] Wimalasiria, G.E.M., Ranasinghe, P., Gunaratnec, D.M.A. and Vidhana Arachchi, L.P. (2016) Antioxidant and Anti-Diabetic Properties of Caryotaurens (Kithul) Flour. Procedia Food Science, 6, 181-185.

[26] Atarzadeh, F., Jaladat, A.M., Dastgheib, L., Amin, G., Nimrouzi, M. and Kamaline- 
jad, M. (2017) Cassia fistula: A Remedy from Traditional Persian Medicine for Treatment of Cutaneous Lesions of Pemphigus vulgaris. Avicenna Journal of Phytomedicine, 7, 107-115.

[27] Aher, A.N., Pal, S.C., Patil, U.K., Yadav, S.K. and Bhattacharya, S. (2009) Evaluation of Antihistaminic Activity of Casuarina equisetifolia Frost (Casuarinaceae). Pharmacology Online, 1, 1144-1149.

[28] Dzeufiet, P.D., Ohandja, D.Y., Tédong, L., Asongalem, E.A., Dimo, T., Sokeng, S.D. and Kamtchouing, P. (2007) Antidiabetic Effect of Ceiba pentandra Extract on Streptozotocin-Induced Non-Insulin-Dependent Diabetic (NIDDM) Rats. African Journal of Traditional, Complementary and Alternative Medicines, 4, 47-54.

[29] Lima, E.B.C., Sousa, C.N.S., Meneses, L.N., Ximenes, N.C., Santos Júnior, M.A., Vasconcelos, G.S., Lima, N.B.C., Patrocínio, M.C.A., Macedo, D. and Vasconcelos, S.M.M. (2015) Cocos nucifera (L.) (Arecaceae): A Phytochemicals and Pharmacological Review. Brazilian Journal of Medical and Biological Research, 48, 953-964. https://doi.org/10.1590/1414-431X20154773

[30] Anwar, F., Latif, S., Ashraf, M. and Gilani, A.H. (2007) Moringa oleifera: A Food Plant with Multiple Medicinal Uses. Phytotherapy Research, 21, 17-25. https://doi.org/10.1002/ptr.2023

[31] Gupta, R. and Das Gupta, G. (2015) A Review on Plant Cordia obliqua Willd. (Clammy Cherry). Pharmacognosy Reviews, 9, 127-131. https://doi.org/10.4103/0973-7847.162124

[32] Mariana, M.G.P., Sidnei, B.O.F., Catarina, E.F., Fábio, B. and Patrícia, D.F. (2013) Anti-Inflammatory Activity of Ethanol Extract and Fractions from Couroupita guianensis Aublet Leaves. Journal of Ethnopharmacology, 146, 324-330. https://doi.org/10.1016/j.jep.2012.12.053.

[33] Rahman, M., Hasan, N., Das, A.K., Hossain, T., Jahan, R., Khatun, A. and Rahmatullah, M. (2011) Effect of Delonix Regia Leaf Extract on Glucose Tolerance in Glucose-Induced Hyperglycemic Mice. African Journal of Traditional, Complementary and Alternative Medicines, 8, 34-36.

[34] Eisa, M.M., Almagboul, A.Z., Omer, M.E.A. and Elegami, A.A. (2000) Antibacterial Activity of Dichrostachys cinera. Fitoterapia, 71, 324-327.

[35] Zhao, T., Sun, Q., Marques, M. and Witcher, M. (2015) Anticancer Properties of Phyllanthus emblica (Indian Gooseberry). Oxidative Medicine and Cellular Longevity, 1, 1-17.

[36] Rayane, C.P., Paula, V.L., Stephanie, C.L., Gleice, K.A.M., Arnildo, P., Marcelo, A.A., Claudio, S.L.B. and Ricardo, A.A.L.(2017) Enterolobium contortisiliquum Is a Cause of Acute Ruminal Acidosis in Sheep. Toxicon, 126, 90-95.

https://doi.org/10.1016/j.toxicon.2016.12.015

[37] Suriyavathana, M., Subha, P., Senthilkumar, S., Sumathi, S., Kavitha, R. and Ramalingam, K. (2016) Phytochemical Profile of Erythrina variegata by Using High-Performance Liquid Chromatography and Gas Chromatography Mass Spectroscopy Analyses. Journal of Acupuncture and Meridian Studies, 9, 207-212. https://doi.org/10.1016/j.jams.2016.06.001

[38] Shukla, R., Sharma, S.B., Puri, D., Prabhu, K.M. and Murthy, P.S. (2000) Medicinal Plants for Treatment of Diabetes Mellitus. Indian Journal of Clinical Biochemistry, 15, 169-177. https://doi.org/10.1007/BF02867556

[39] Sackeyfio, A.C. and Lugeleka, O.M. (1986) The Anti-Inflammatory Effect of a Crude Aqueous Extract of the Root Bark of "Ficus elastic" in the Rat. Archives Internationales de Pharmacodynamie et de Thérapie, 1, 169-176. 
[40] Kar, A., Choudhary, B.K. and Bandyopadhyay, N.G. (2003) Comparative Evaluation of Hypoglycaemic Activity of Some Indian Medicinal Plants in Alloxan Diabetic Rats. Journal of Ethnopharmacology, 84, 105-108.

[41] Singh, D., Singh, B. and Goel, R.K. (2011) Traditional Uses, Phytochemistry and Pharmacology of Ficus religiosa: A Review. Journal of Ethnopharmacology, 134, 565-583.

[42] Simonsen, H.T., Nordskjold, J.B., Smitt, U.W., Nyman, U., Palpu, P., Joshi, P. and Varughese, G. (2001) In Vitro Screening of Indian Medicinal Plants for Antiplasmodial Activity. Journal of Ethnopharmacology, 2, 195-204.

[43] Sharma, M., Chopra, S. and Prasad, S.B. (2015) Guazuma tomentosa: A Valuable Medicinal Plant. International Journal of Pharmacognosy and Phytochemical Research, 7, 197-200.

[44] Vanzella, C., Bianchetti, P., Sbaraini, S., Vanzin, S.I., Melecchi, M.I.S., Caramão, E.B. and Siqueira, I.R. (2012) Antidepressant-Like Effects of Methanol Extract of Hibiscus tiliaceus Flowers in Mice. BMC Complementary and Alternative Medicine, No. 41, 1-6. https://doi.org/10.1186/1472-6882-12-41

[45] Sastri (1959) Ixora pavetta. http://plants.jstor.org/compilation/ixora.pavetta

[46] Kumar, S., Kumar, V. and Prakash, O.M. (2012) Antidiabetic and Hypolipidemic Activities of Kigelia pinnata Flower Extract in Streptozotocin Induced Diabetic Rats. Asian Pacific Journal of Tropical Biomedicine, 2, 543-546.

[47] Vedavathy, S., Sudhakar, A. and Mrdula, V. (1997) Tribal Medicinal Plants of Chittoor. Ancient Science of Life, 16, 307-331.

[48] Sinha, J., Singh, V., Singh, J. and Rai, A.K. (2017) Phytochemistry, Ethnomedical Uses and Future Prospects of Mahua (Madhuca longifolia) as a Food: A Review. Journal of Nutrition and Food Sciences, 7, 573-580. https://doi.org/10.4172/2155-9600.1000573

[49] Shah, K.A., Patel, M.B., Patel, R.J. and Parmar, P.K. (2010) Mangifera Indica (Mango). Pharmacognosy Reviews, 4, 42-46. https://doi.org/10.4103/0973-7847.65325

[50] Banzouzi, J.T., Proust, A., Rajemiarimiraho, M. and Ongoka, P. (2008) Traditional Uses of the African Millettia Species (Fabaceae). International Journal of Botany, 4, 406-420. https://doi.org/10.3923/ijb.2008.406.420

[51] Babitha, S., Banji, D. and Banji, O.J.F. (2012) Antioxidant and Hepatoprotective Effects of Flower Extract of Millingtonia hortensis Linn. on Carbon Tetrachloride Induced Hepatotoxicity. Journal of Pharmacy and Bioallied Sciences, 4, 307-312. https://doi.org/10.4103/0975-7406.103258

[52] Gami, B., Pathak, S. and Parabia, M. (2012) Ethnobotanical, Phytochemical and Pharmacological Review of Mimusops elengi Linn. Asian Pacific Journal of Tropical Biomedicine, 2, 743-748.

[53] Agrawal, J. and Pal, A. (2013) Nyctanthes arbortristis Linn-A Critical Ethnopharmacological Review. Journal of Ethnopharmacology, 146, 645-658.

[54] Millogo-Kone, H., Guissou, I.P., Nacoulma, O. and Traore, A.S. (2007) Antimicrobial Effects of the Stem Bark Extracts of Parkia Biglobosa (Jacq.) Benth. on Shigellae. African Journal of Traditional, Complementary and Alternative Medicines, 4, 392-396. https://doi.org/10.4314/ajtcam.v4i4.31234

[55] Subramanian, R., Subbramaniyan, P. and Raj, V. (2015) Isolation of Bergenin from Peltophorum pterocarpum Flowers and Its Bioactivity. Beni-Suef University Journal of Basic and Applied Sciences, 4, 256-261.

https://doi.org/10.1016/j.bjbas.2015.06.002 
[56] Rahmani, A.H., Aly, S.M., Ali, H., Babiker, A.Y., Srikar, S. and Khan, A.A. (2014) Therapeutic Effects of Date Fruits (Phoenix dactylifera) in the Prevention of Diseases via Modulation of Anti-Inflammatory, Anti-Oxidant and Anti-Tumor Activity. International Journal of Clinical and Experimental Medicine, 7, 483-491.

[57] Lim, T.K. (2013) Edible Medicinal and Non Medical Plants. Vol. 7, 87-88.

[58] Hamburger, M.O., Cordell, G.A. and Ruangrungsi, N. (1991) Traditional Medicinal Plants of Thailand. XVII. Biologically Active Constituents of Plumeria rubra. Journal of Ethnopharmacology, 31, 5-8.

[59] Katkar, K.V., Suthar, A.C. and Chauhan, V.S. (2010) The Chemistry, Pharmacologic, and Therapeutic Applications of Polyalthia longifolia. Pharmacognosy Reviews, 4, 62-68. https://doi.org/10.4103/0973-7847.65329

[60] Brijesh, S., Daswani, P.G., Tetali, P., Rojatkar, S.R., Antia, N.H. and Birdi, T.H. (2006) Studies on Pongamia pinnata (L.) Pierre Leaves: Understanding the Mechanism(s) of Action in Infectious Diarrhea. Journal of Zhejiang University Science B, 7, 665-674. https://doi.org/10.1631/jzus.2006.B0665

[61] Joseph, B. and Priya, R.M. (2011) Phytochemical and Biopharmaceutical Aspects of Psidium guajava (L.) Essential Oil: A Review. Research Journal of Medicinal Plants, 5, 432-442. https://doi.org/10.3923/rjmp.2011.432.442

[62] Ghalem, B.R. and Mohamed, B. (2008) Antibacterial Activity of Leaf Essential Oils of Eucalyptus globulus and Eucalyptus camaldulensis. African Journal of Pharmacy and Pharmacology, 2, 211-215.

[63] Paramaguru, R., Mazumder, P.M., Sasmal, D. and Jayaprakash, V. (2014) Ant Diabetic Activity of Pterospermum acerifolium Flowers and Glucose Uptake Potential of Bioactive Fraction in L6 Muscle Cell Lines with Its HPLC Fingerprint. BioMed Research International, 1, 1-11. https://doi.org/10.1155/2014/459376

[64] Ravishankara, M.N., Pillai, A.D., Padh, H. and Rajani, M. (2003) A Sensitive HPTLC Method for Estimation of Amentoflavone, a Bioactive Principle from Biophytum sensitivum (Linn.) DC. and Putranjiva roxburghii Wall. Journal of Planar Chromatography-Modern TLC.

[65] Pearson, M.N., Thomas, J.E. and Randies, J.W. (1994) Detection of an Unidentified Potyvirus from Roystonea regia Palm Using the Polymerase Chain Reaction and Degenerate, Potyvirus Specific, Primers and Potential Problems Arising from the Amplification of Host Plant DNA Sequences. Journal of Virological Methods, 50, 211-217. https://doi.org/10.1016/0166-0934(94)90177-5

[66] Arumugam, S., Selvaraj, S.V., Velayutham, S., Natesan, S.K. and Palaniswamy, K. (2011) Evaluation of Anti-Ulcer Activity of Samanea saman (Jacq) Merr Bark on Ethanol and Stress Induced Gastric Lesions in Albino Rats. Indian Journal of Pharmacology, 43, 586-590. https://doi.org/10.4103/0253-7613.84978

[67] Tripathi, Y.C., Prabhu, V.V., Pal, R.S. and Mishra, R.N. (1996) Medicinal Plants of Rajasthan in Indian System of Medicine. Ancient Science of Life, 15, 190-212.

[68] Rachel, K.V., Vimala, Y. and Apta Chaitanya, D. (2013) A Trypsin Inhibitor-SNTI with Anti Dandruff Activity from Sapindus trifoliatus. Indian Journal of Applied Research, 3, 3-5. https://doi.org/10.15373/2249555X/MAR2013/2

[69] Nataraj, H.R. and Hiremath, K. (2009) Pharmacognostic and Phytochemical Analysis of Different Market Samples of Ashoka (Saraca indica Linn). Ancient Science of Life, 29, 7-11.

[70] Makinde, J.M., Amusan, O.O.G. and Adesogan, E.K. (1988) The Ant Malarial Activity of Spathodea campunulata Stems Bark Extract on Plasmodium berghei Berg- 
hei in Mice. Phytotherapy Research, 4, 53-56.

https://doi.org/10.1002/ptr.2650040204

[71] Chandra, P.K. (2009) Aboriginal Uses and Management of Ethnobotanical Species in Deciduous Forests of Chhattisgarh State in India. Journal of Ethnobiology and Ethno Medicine, 5, 1-9.

[72] Singh, N.P. and Singh, V.K. (1976) Streblus asper Lour-An Ancient Indian Drug for Cure of Filariasis. Acta Botanica Indica, 15, 108-109.

[73] Muruganandan, S., Srinivasan, K., Chandra, S., Tandan, S.K., Lal, J. and Raviprakash, V. (2001) Anti-Inflammatory Activity of Syzygium cumini Bark. Fitoterapia, 72, 369-375.

[74] Agarwal, M. and Chauhan, S. (2015) Anti-Mycobacterial Potential of Tabebuia aurea (Manso) Benth \& Hook (Bignoniaceae). Journal of Medicinal Plants Studies, 3, 63-68.

[75] Julia, M.F. (1958) Tamarind (Tamarindus indica L.) Its Food, Medicinal and Industrial Uses. Florida State Horticultural Society, 288.

[76] Majumdar, M., Nayeem, N., Kamath, J.V. and Asad, M. (2007) Evaluation of Tectona grandis Leaves for Wound Healing Activity. Pakistan Journal of Pharmaceutical Sciences, 20, 120-124.

[77] Dwivedi, S. (2007) Terminalia arjuna Wight and Arn.-A Useful Drug for Cardiovascular Disorders. Journal of Ethnopharmacology, 1, 114-129.

[78] Khan, A.A., Kumar, V., Singh, B.K. and Singh, R. (2014) Evaluation of Wound Healing Property of Terminalia catappa on Excision Wound Models in Wistar Rats. Drug Res (Stuttg).

[79] Viswanatha, G.L., Srinath, R., Nandakumar, K., Shylaja, H. and Lakshman, K. (2007) Antidiarrheal Activity of Alcoholic and Aqueous Extracts of Stem Bark of Thespesia populnea in Rodents. Pharmacology Online, 3, 222-230.

[80] Shruthi, A., Latha, K.P., Vagdevi, H.M., Pushpa, B. and Shwetha, C. (2012) Anti-Diabetic Activity of the Leaves Extracts of Wrightia tinctoria on Alloxan Induced Diabetic Rats. Journal of Chemical and Pharmaceutical Research, 4, 3125-3128.

[81] Tahergorabi, Z., Abedini, M.R., Mitra, M., Fard, M.H. and Beydokhti, H. (2015) "Ziziphus jujuba": A Red Fruit with Promising Anticancer Activities. Pharmacognosy Reviews, 9, 99-106. https://doi.org/10.4103/0973-7847.162108

[82] Reddy, A.K., Joy, J.M. and Kumar, A. (2011) Lannea coromandelica: The Researcher's Tree. Journal of Pharmacy Research, 4, 577-579.

[83] Angeline, A.O., Lawrence, A.O.M., Phillip, O.O., Isaac, O.J., Regina, A.N., Hosea, A., Pauline, M. and Sylvia, A.O. (2015) Bioactive Carbazole Alkaloids from Alysicarpus ovalifolius (Schumach). Journal of the Korean Society for Applied Biological Chemistry, 58, 839-846. https://doi.org/10.1007/s1376

[84] Hemendra, S.C. and Sushil, K.S. (2011) Phytochemical Analysis, Antioxidant and Anti-Inflammatory Activities of Phyllanthus simplex. Journal of Ethnopharmacology, 137, 1337-1344. https://doi.org/10.1016/j.jep.2011.07.069

[85] Patel, D.K., Kumar, R., Sairam, K. and Hemalatha, S. (2013) Hybanthus enneaspermus (L.) F. Muell: A Concise Report on Its Phytopharmacological Aspects. Chinese Journal of Natural Medicines, 11, 199-206. https://doi.org/10.1016/S1875-5364(13)60017-5

[86] Imam, H., Sofi, Z.G., Seikh, A. and Lone, A. (2014) The Incredible Benefits of Nagarmotha (Cyperus rotundus). International Journal of Nutrition, Pharmacology, Neurological Diseases, 4, 23-27. https://doi.org/10.4103/2231-0738.124611 
[87] Panda, H. (2002) Medicinal Plants Cultivation \& Their Uses. Asia Pacific Business Press Inc.

[88] http://tropical.theferns.info/viewtropical.php?id=Corchorus+aestuans

[89] Van Thai, H., Kim, E., et al. (2015) Boerhavia diffusa L. Ethanol Extract Suppresses Inflammatory Responses via Inhibition of Src/Syk/TRAF6. Journal of Functional Foods, 17, 476-490.

[90] Sultana, K.W., Chatterjee, S., Roy, A. and Chandra, I. (2015) An Overview on Ethnopharmacological and Phytochemical Properties of Thunbergia sp. Medicinal Aromatic Plants Journals, 4, 217.

[91] Mujeeb, M., Aeri, V., Bagri, P. and Khan, S.A. (2009) Hepatoprotective Activity of the Methanolic Extract of Tylophora indica (Burm. f.) Merill. Leaves. International Journal of Green Pharmacy.

[92] Arunachalam, K. and Parimelazhagan, T. (2012) Antidiabetic Activity of Aqueous Root Extract of Merremia tridentata (L.) Hall. f. in Streptozotocin-Induced-Diabetic Rats. Asian Pacific Journal of Tropical Medicine.

[93] Sunil, K., et al. (2012) Traditional Medicinal Plants Curing Diabetes: A Promise for Today and Tomorrow Traditional Medicinal Plants Curing Diabetes. Asian Journal of Traditional Medicines.

[94] Das, N.J., Saikia, S.P., et al. (2016) Medicinal Plants of North-Kamrup District of Assam Used in Primary Healthcare System. Indian Journal of Traditional Knowledge, 5, 489-493.

[95] Pandi, K.P., Ayyanar, M., et al. (2007) Medicinal Plants Used by Malasar Tribes of Coimbatore District, Tamil Nadu. Indian Journal of Traditional Knowledge, 6, 579-582. https://doi.org/10.3923/rjmp.2011.567.576

[96] Butle, A., Talmale, S. and Patil, M.B. (2016) Potential in Vivo Immunomodulatory Effects of the Most Active Lectin Isolated from Seeds of Zizyphus encolpia. Journal of Clinical and Cellular Immunology, 7, 386.

[97] https://www.bimbima.com/ayurveda/putrajiva-treeputranjiva-roxburghii-medicinal -uses/263/

[98] Vaghasiya, Y., Dave, R. and Chanda, S. (2011) Phytochemical Analysis of Some Medicinal Plants from Western Region of India. Research Journal of Medicinal Plants, 5, 567-576.

[99] Harisha, R.P. and Padmavathy, S. (2013) Knowledge and Use of Wild Edible Plants in Two Communities in Malai Madeshwara Hills, Southern India. International Journal of Botany, 64-72. https://doi.org/10.3923/ijb.2013.64.72

[100] http://www.stuartxchange.org/Malakafe.html

[101] Mishra, N., Oraon, A., et al. (2010) Anticonvulsant Activity of Benkara malabarica (Linn.) Root Extract: In Vitro and in Vivo Investigation. Journal of Ethnopharmacology, 128, 533-536.

[102] http://tropical.theferns.info/viewtropical.php?id=Cissus+verticillata

[103] Jesus, N.Z.T., FalcÃ£o, H.S., et al. (2013) Hyptis suaveolens (L.) Poit (Lamiaceae), a Medicinal Plant Protects the Stomach against Several Gastric Ulcer Models. Journal of Ethnopharmacology, 150, 982-988.

[104] Shang-Chih Lai, D.C., et al. (2010) Antioxidant and Antiproliferative Activities of Desmodium triflorum (L.). The American Journal of Chinese Medicine, 38, 329. https://doi.org/10.1142/S0192415X10007889

[105] Salatino, A., Salatino, M.L.F. and Giuseppina, N.J. (2007) Traditional Uses, Chemi- 
stry and Pharmacology of Croton Species (Euphorbiaceae). Journal of the Brazilian Chemical Society, 18.

[106] Food, P.S. (1996) Pharmaceutical and Industrial Potential of Carissa genus. An Overview. Reviews in Environmental Science and Biol Technology.

[107] Jayakumar, S. and Jhancy, M., et al. (2010) Evaluation of Antioxidant Potential and Antibacterial Activity of Calotropis gigantean and Vinca rosea using in Vitro Model. Indian Journal of Science and Technology, 720-723. 